\title{
New Method to Calculate Spatial Distribution of Acousto-Optic Figure of Merit in Crystals
}

\author{
A.V. Muromets ${ }^{a *}$ And A.S. Trushin ${ }^{b}$ \\ ${ }^{a}$ Department of Physics, Lomonosov Moscow State University, Moscow, 119991, Russia \\ ${ }^{b}$ Lebedev Physical Institute of RAS, Leninsky Prospect 53, Moscow, 119991, Russia
}

\begin{abstract}
We propose a novel method to calculate acousto-optic figure of merit in crystals. Calculations are performed in laboratory coordinate system where $Z^{\prime}$-axis is collinear with wave vector of ultrasound and the Fresnel equation is considered as an equation on the third component of refractive index vector. The method is applicable to both uniaxial and biaxial crystals. In this paper, we compared obtained values of acousto-optic figure of merit with values from literature data for uniaxial crystals such as paratellurite, lithium niobate, tellurium and for biaxial crystals such as lead and strontium tetraborates. Calculations in paratellurite were carried out for slow-shear acoustic wave propagating along [110] crystal axis. In lithium niobate crystal, we perform comparison with results for geometry of acousto-optic interaction where acoustic wave vector forms $88^{\circ}$ angle with $X$ crystal axis and $150.4^{\circ}$ angle with $Z$ crystal axis. In tellurium crystal, we investigate geometries applied in infrared deflectors. In $\mathrm{SrB}_{4} \mathrm{O}_{7}$ and $\mathrm{PbB}_{4} \mathrm{O}_{7}$ crystals we analyze acousto-optic characteristics of slow-shear mode propagating along [100] crystal axis. Spatial distributions of acousto-optic figure of merit and acoustic frequency for the mentioned acousto-optic interaction geometries are presented.
\end{abstract}

DOI: $10.12693 /$ APhysPolA.127.93

PACS: 78.20.hb, 42.79.Jq, 43.35.Cg, 62.30.+d, 46.15.-x, 78.20.Bh

\section{Introduction}

Acousto-optic (AO) devices are widely used in image processing, laser spectroscopy, optoelectronics, medicine, etc. [1-3]. These devices control characteristics of a laser beam diffracted on gratings generated by ultrasound in crystals. Intensity of diffracted light depends on acoustic driving power and $\mathrm{AO}$ figure of merit (AOFM) $M_{2}$. In contemporary AO devices, planes of light and ultrasound interaction are limited to basic crystal planes, which is not optimal. In order to find optimal geometries of AO interaction, it is necessary to develop an effective method of precise and fast calculation of $M_{2}$ coefficient for arbitrary directions of light and ultrasound. In addition, selection of optimal $M_{2}$ values for ordinary and extraordinary light beams propagating along arbitrary directions provides an opportunity to design AO devices not sensitive to polarization of incident light $[4,5]$.

\section{Calculation method}

In a crystalline media, AOFM is determined by the expression $[1,4]$ :

$$
\begin{aligned}
& M_{2}=\frac{1}{4 n_{0} n_{1} \rho V^{3}} \\
& \quad \times\left[d_{i}^{(1)} \varepsilon_{i j} \varepsilon_{k l} d_{l}^{(0)} p_{j k m n}\left(r_{m} m_{n}+r_{n} m_{m}\right)\right]^{2},
\end{aligned}
$$

where $n_{0}$ and $n_{1}$ - refractive indices of incident and diffracted plane waves, respectively, $\varepsilon_{i j}-$ components of dielectric permittivity tensor, $d_{l}^{(0)}$ and $d_{i}^{(1)}-$ corresponding components of a unit electric displacement field

\footnotetext{
*corresponding author; e-mail: anastasiagirl@mail.ru
}

vector for incident and diffracted optic waves, $p_{j k m n}$ photoelastic tensor components, $r_{m}$ and $m_{n}$ - components of acoustic polarization and wave normal vectors, $\rho$ - medium density and $V$ - phase velocity of ultrasound. Polarization direction $r$ and phase velocity $V$ of an acoustic wave can be found by solving the Christoffel elastodynamic equation [1]:

$$
\left(c_{i j k l} m_{j} m_{k}-\rho V^{2} \delta_{i l}\right) r_{i}=0,
$$

where $c_{i j k l}$ - components of elastic tensor, and $\delta$ - the Kronecker delta symbol.

Directions of incident and diffracted light are determined according to momentum conservation law

$$
\boldsymbol{k}_{1}=\boldsymbol{k}_{0}+\boldsymbol{K}
$$

where $\boldsymbol{k}_{1}$ and $\boldsymbol{k}_{0}$ - wave vectors of diffracted and incident light, correspondingly, $\boldsymbol{K}$ - wave vector of ultrasound. Optic wave polarization and refraction index can be found by solving the Fresnel equation [6]:

$$
\left(n^{2} \delta_{i j}-n_{i} n_{j}-\varepsilon_{i j}\right) e_{j}=0,
$$

where $n$ - absolute value of refractive index vector $\boldsymbol{n}$ with the components $n_{i}$ and $n_{j}, e_{j}$ - components of electric field related to the electric displacement field components described by the relation $d_{i}=\varepsilon_{i j} e_{j}$.

Equation (3) can be solved analytically for the case of isotropic or optical uniaxial media. This approach was applied in the papers [7-9] for particular geometries of AO interaction. Unfortunately, the used method of analysis is not applicable to biaxial crystals. In our approach, we use for calculations laboratory coordinate system $\left(X^{\prime} Y^{\prime} Z^{\prime}\right)$ where $Z^{\prime}$-axis is collinear with ultrasound wave vector direction. The Fresnel Eq. (4) is considered as an equation on the third component $n_{z^{\prime}}^{\prime}$ of refractive index vector while first two components $\left(n_{x^{\prime}}^{\prime}, n_{y^{\prime}}^{\prime}\right)$ are assumed to be known. Data in Fig. 1a represents refractive index surfaces for ordinary and extraordinary 
light propagating in an anisotropic (for example, uniaxial) crystal in the transformed coordinate system where acoustic wave vector $\boldsymbol{m}$ becomes vertical. Intersections of the vertical line with the refractive index surfaces give directions of the incident and diffracted light, as shown in Fig. 1b. After multiplication by the scaling factor $2 \pi / \lambda$, we obtain the wave vectors which satisfy the momentum law in Eq. (3). The length of the acoustic wave vector determines the magnitude of acoustic frequency.

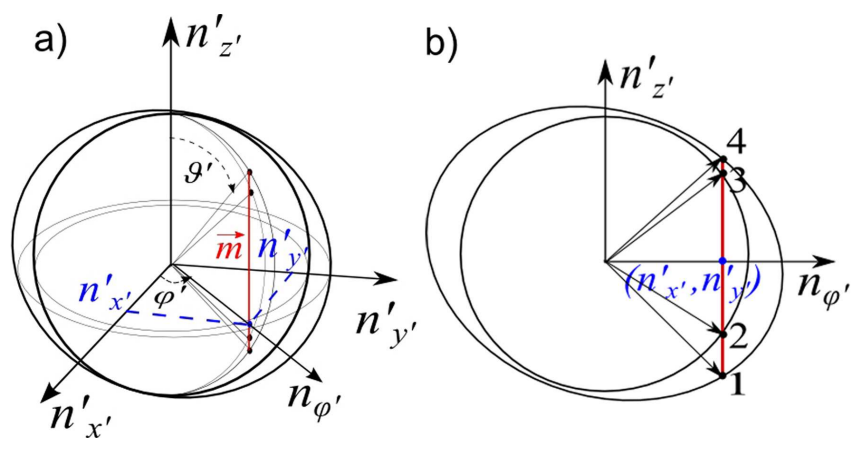

Fig. 1. (a) Scheme of AO interaction in laboratory coordinate system. (b) Cut of refractive index surfaces crossed by acoustic vector with a number of intersections.

In case of biaxial crystals, it is not possible to obtain an analytical expression describing propagation direction of the diffracted light. It should be mentioned that generic algorithms providing solution of non-linear equations require several dozen iterations in order to solve the Fresnel equation with a tolerable accuracy. On the other hand, in the proposed method, a single iteration in solving the Fresnel equation gives us information on the optic and acoustic wave vectors satisfying momentum conservation law, on the polarizations of the incident and diffracted light as well as on the frequency of ultrasound. It means that the new method is several dozen times faster and it is applicable to both uniaxial and biaxial crystals.

\section{Results}

Paratellurite crystal is very important for $\mathrm{AO}$ in the visible range due to the high $M_{2}$ value. In Fig. 2 spatial distributions of the figure of merit $M_{2}(\theta, \varphi)$ and the ultrasound frequency $f(\theta, \varphi)$ are shown at the [110] direction of ultrasound propagation. In the figure, $\theta$ and $\varphi$ - polar and azimuthal angles of the crystal coordinate system. We performed the calculation without consideration of optical activity, piezoelectric effect and assuming linear polarization of incident and diffracted light. Therefore the maximal value $M_{2} \approx 800 \times 10^{-15} \mathrm{~s}^{3} / \mathrm{kg}$, which agrees with literature data $[10,11]$.

Lithium niobate $\left(\mathrm{LiNbO}_{3}\right)$ demonstrates good $\mathrm{AO}$ properties and strong piezoelectric effect. This crystal is widely used in acousto-optic, electro-optic and nonlinearoptic devices, high-frequency piezoelectric transducers, delay lines, etc. [1-5]. Elastic constants in Eq. (2) were "stiffed" in order to take piezoelectric effect into account. We compared calculated $M_{2}$ values for $13^{\circ}-Z$ cut of
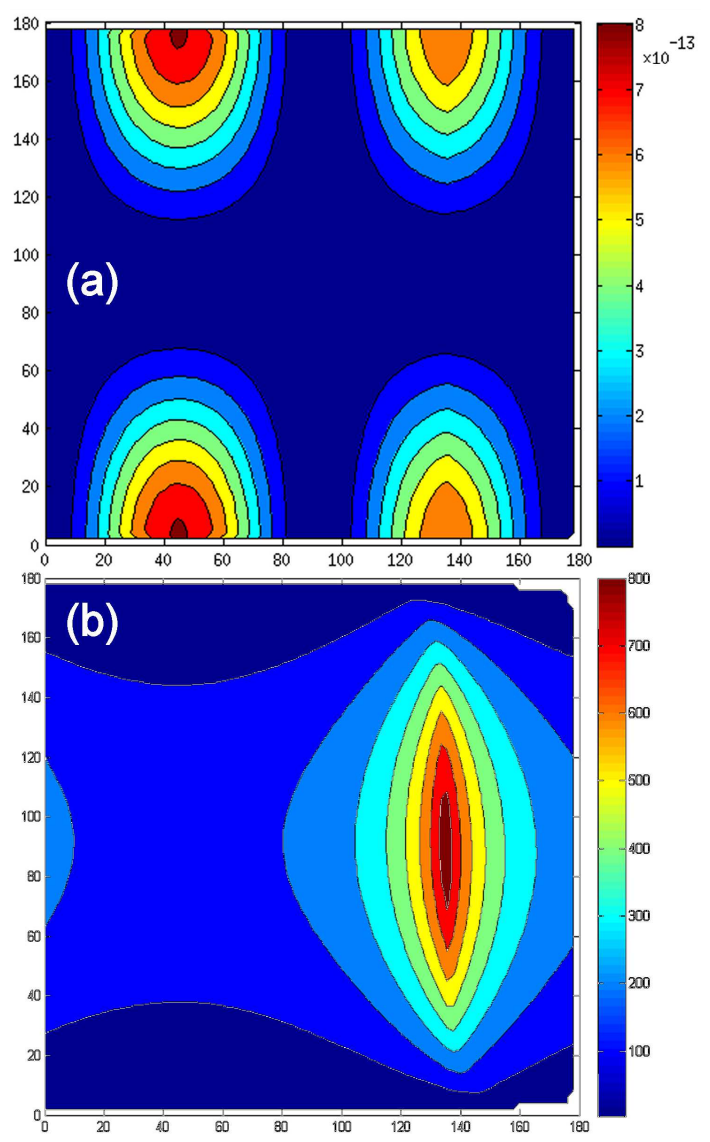

Fig. 2. Spatial distributions of figure of merit $M_{2}(\theta, \varphi) \mathrm{s}^{3} / \mathrm{kg}$ (a) and frequency $f(\theta, \varphi) \mathrm{MHz}$ (b) for the acoustic mode with $V=617 \mathrm{~m} / \mathrm{s}$ in $\mathrm{TeO}_{2}$ crystal, acoustic wave direction [110], anisotropic diffraction.

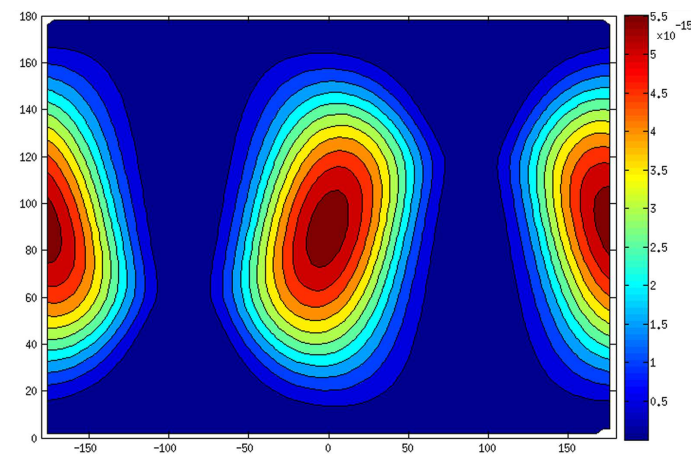

Fig. 3. Spatial distributions of figure of merit $M_{2}(\theta, \varphi) \mathrm{s}^{3} / \mathrm{kg}$ for the acoustic mode with $V=$ $3986 \mathrm{~m} / \mathrm{s}$ in $\mathrm{LiNbO}_{3}$ crystal, $\left(\theta_{a}, \varphi_{a}\right)=\left(150.4^{\circ}, 88^{\circ}\right)$, isotropic diffraction $(\mathrm{o} \rightarrow \mathrm{o})$.

$\mathrm{LiNbO}_{3}$ with values known from literature $[4,5]$. This cut was thoroughly investigated in mentioned literature because of close values of AOFM for two optical polarizations (ordinary and extraordinary). Spatial distribution $M_{2}(\theta, \varphi)$ is shown in Fig. 3 for the direction of ultrasound wave vector propagation $\left(\theta_{a}, \varphi_{a}\right)=\left(150.4^{\circ}, 88^{\circ}\right)$, where $\theta_{a}$ - angle between acoustic wave vector and $Z$ crystal axis and $\varphi_{a}$ - angle between acoustic wave vector and 


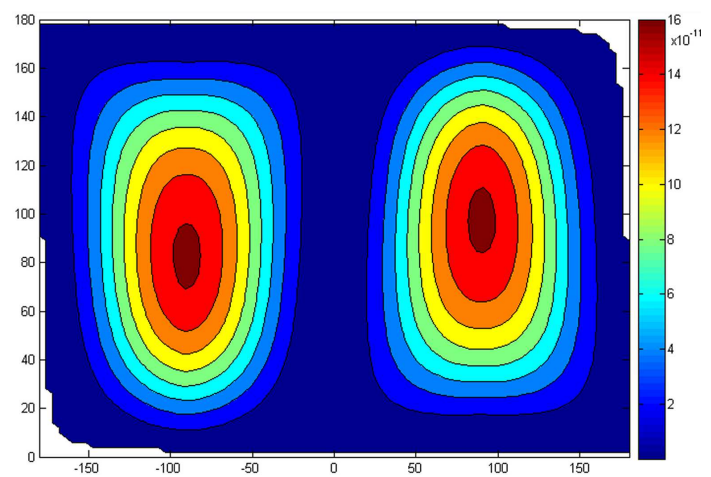

Fig. 4. As Fig. 3 with $V=974 \mathrm{~m} / \mathrm{s}$ in Te crystal, acoustic wave direction [100], anisotropic diffraction.
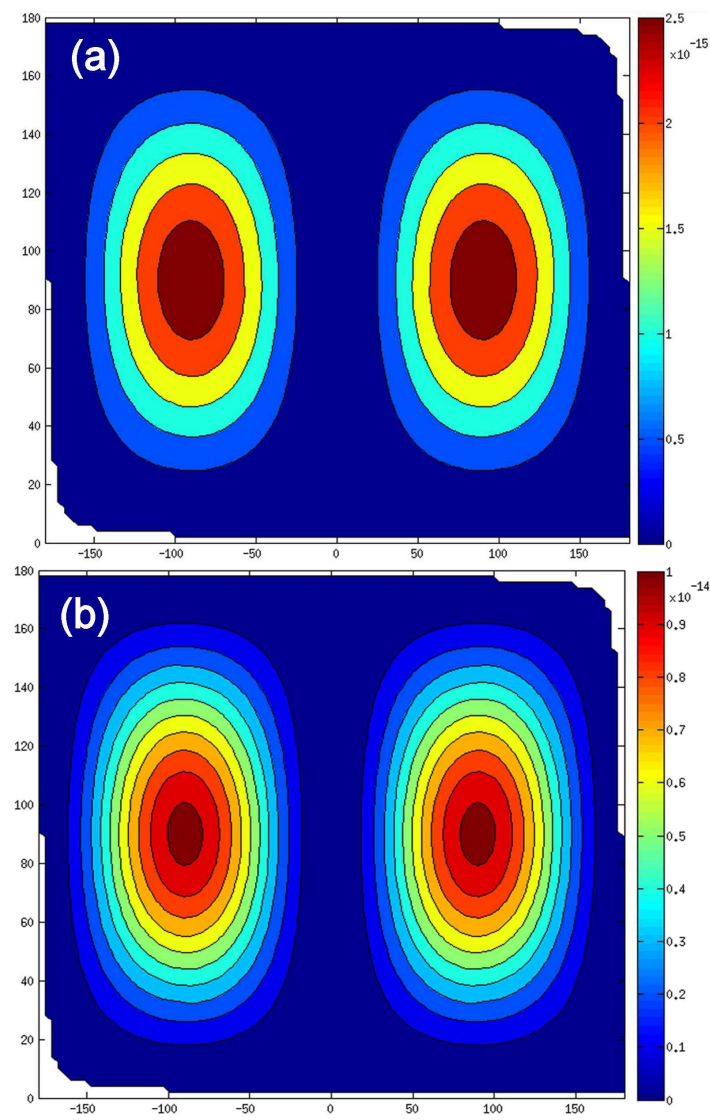

Fig. 5. As Fig. 3 with (a) $V=4480 \mathrm{~m} / \mathrm{s}$ in $\mathrm{PbB}_{4} \mathrm{O}_{7}$ (a) and $V=5470 \mathrm{~m} / \mathrm{s}$ in $\mathrm{SrB}_{4} \mathrm{O}_{7}(\mathrm{~b})$, acoustic wave direction [100], anisotropic diffraction.

$X$ crystal axis. The maximal magnitude of $M_{2}$ for the diffraction from ordinary to ordinary light wave is close to the magnitude known from the article [9].

Tellurium (Te) crystal is interesting as a material for AO devices operating in the far infrared range of electromagnetic spectrum. Our calculations were carried out without consideration of the piezoelectric effect due to its low influence on the acoustic wave velocities and polarizations [12]. The obtained spatial distribution of $M_{2}(\theta, \varphi)$ for the direction [100] of the longitudinal ultrasonic wave propagation is shown in Fig. 4. The presented values of $M_{2}$ are in close agreement with data in Ref. [7].

Lead and strontium tetraborates $\mathrm{PbB}_{4} \mathrm{O}_{7}$ and $\mathrm{SrB}_{4} \mathrm{O}_{7}$ are promising $\mathrm{AO}$ materials because of the high value of AOFM [13]. Maximal AOFM values for $\mathrm{PbB}_{4} \mathrm{O}_{7}$ and $\mathrm{SrB}_{4} \mathrm{O}_{7}$ in mentioned article are in good correspondence with obtained values represented in Fig. 5. Calculations in both crystals were carried out for slow-shear mode propagating along [100] crystal axis with polarization along [001] crystal axis.

\section{Conclusions}

A fast and precise method of AOFM calculation is proposed. The method allows calculating spatial distributions of AOFM and acoustic frequency for both uniaxial and biaxial crystals. Results obtained with the new method for crystals $\mathrm{TeO}_{2}, \mathrm{LiNbO}_{3}, \mathrm{Te}, \mathrm{SrB}_{4} \mathrm{O}_{7}$ and $\mathrm{PbB}_{4} \mathrm{O}_{7}$ are in a good agreement with literature data which confirms validity and applicability of the proposed calculation method.

\section{Acknowledgments}

The research was supported by the grant of the Russian Science Foundation (RSCF, grant no. 14-12-00380).

\section{References}

[1] V.I. Balakshy, V.N. Parygin, L.E. Chirkov, Physical Fundamentals of Acousto-Optics, Radio i Svyaz, Moscow 1985 (in Russian).

[2] J. Xu, R. Stroud, Acousto-Optic Devices, Wiley, New York 1992

[3] A. Goutzoulis, D. Pape, Design and Fabrication of Acousto-Optic Devices, Marcel Dekker, New York 1994.

[4] L.I. Mikheev, V.I. Balakshy, in: Physics and Mathematics, Kazan. Gos. Univ. Uchen. Zap. Ser. Fiz. Mat. Nauki 155, 113 (2013) (in Russian).

[5] V.I. Balakshy, V.B. Voloshinov, V.V. Solodovnikov, in: Proc. Scientific-Practical Conf. "Fundamental and Applied Aspects of Innovative Projects at the Physics Department of MSU", Moscow 11, 173 (2009).

[6] L.D. Landau, E.M. Lifshitz, Electrodynamics of Continuous Media, Butterworth-Heinemann, Oxford 1984.

[7] V.B. Voloshinov, V.I. Balakshy, L.A. Kulakova, N. Gupta, J. Opt. A Pure Appl. Opt. 10, 095002 (2008).

[8] A.S. Andrushchak, E.M. Chernyhivsky, Z.Yu. Gotra, M.V. Kaidan, A.V. Kityk, N.A. Andrushchak, T.A. Maksymyuk, B.G. Mytsyk, W. Schranz, J. Appl. Phys. 108, 103118 (2010).

[9] O.A. Buryy, A.S. Andrushchak, O.S. Kushnir, S.B. Ubizskii, D.M. Vynnyk, J. Appl. Phys. 113, 083103 (2013).

[10] N. Uchida, N. Niizeki, Proc. IEEE 61, 8 (1973).

[11] N. Uchida, Y. Ohmachi, J. Appl. Phys. 40, 12 (1969).

[12] N.V. Polikarpova, P.V. Malneva, Bull. Russ. Acad. Sci. Phys. 76, 12 (2012).

[13] I. Martynyuk-Lototska, T. Dudok, O. Mys, R. Vlokh, Opt. Mater. 31, 4 (2009). 